Supplementary information

\title{
A Helix-Stabilizing Linker Improves Subcutaneous Bioavailability of a Helical Peptide Independent of Linker Lipophilicity
}

Liang Zhang", Tejas Navaratna, and Greg M. Thurber ${ }^{* \#}$

\section{Table of Contents}

1. Mechanistic Interpretation of Results

2. Mass Spectrometry Characterization

3. HPLC Purity and Characterization

4. Protease Digest Data

5. In Vivo Stability Data

6. Model and Fits of Subcutaneous Delivery

7. $\log \mathrm{D}$ data

8. Non-specific cellular uptake 


\section{Mechanistic Interpretation of Results}

\section{Hydrophilic Lipophilic}

\section{Stabilized}

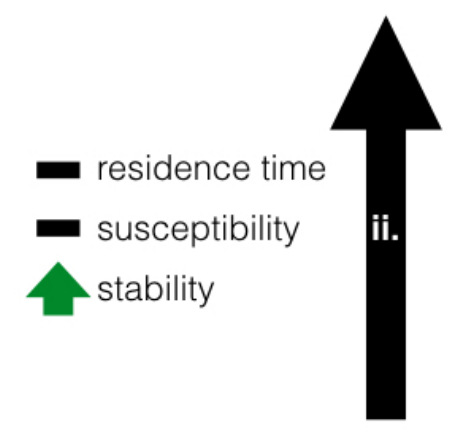

\section{Unstabilized}
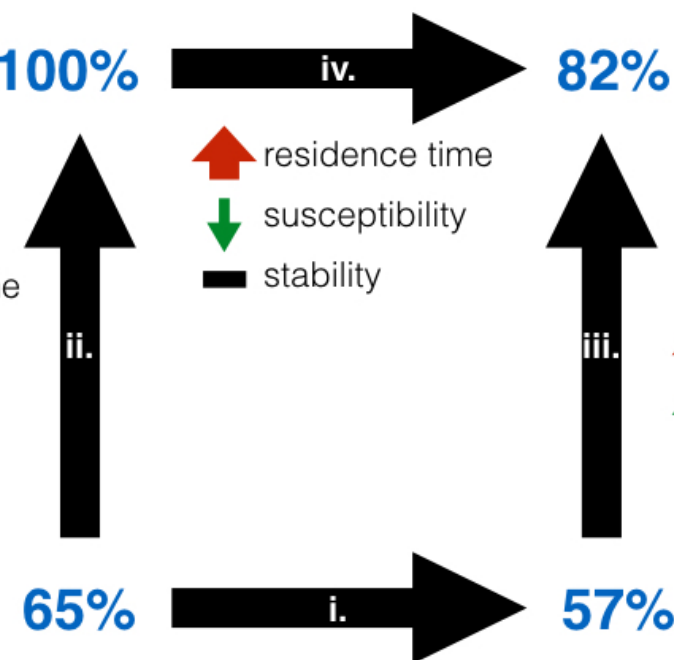

stability

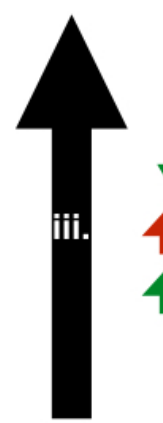

residence time

susceptibility

stability

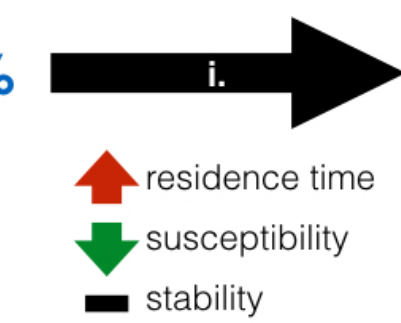

Figure S1. Mechanistic interpretation of results. Based on the experimental results, the above diagram summarizes the proposed mechanistic interpretation of the results. The stabilization chemistry and lipophilicity of the dye on the linker impact the residence time in the skin (i.e. absorption rate), the susceptibility of the molecule in the skin to proteases, and the protease stability of the peptide itself. These three factors can explain the overall trends in bioavailability. Increasing the lipophilicity of the dye for the unstabilized peptides increases the residence time in the skin, but it also lowers the susceptibility of the peptides to be degraded presumably by increasing binding to proteins. These mechanisms effectively cancel out to result in no statistically significant change in bioavailability (i.). The helix-stabilizing linker increases protease stability in both cases, driving an increase in bioavailability (ii. and iii.), but the lower protein binding of the lipophilic stabilized peptide versus the unstabilized peptide (possibly caused by the short PEG linker) increases the susceptibility to protease degradation in the skin. The net result is a reduced positive impact on bioavailability (iii.) relative to stabilization of the hydrophilic peptides (ii.). Increasing the lipophilicity of the stabilized peptide significantly increases the residence time but lowers the susceptibility to a lesser extent, resulting in lower bioavailability (iv.). Although increased lipophilicity tends to slow absorption and lower susceptibility to skin proteases (i. and iv.), the relative magnitude of these effects does not necessarily cancel out and the net result can increase or decrease the overall bioavailability. 


\section{Mass Spectrometry Characterization}
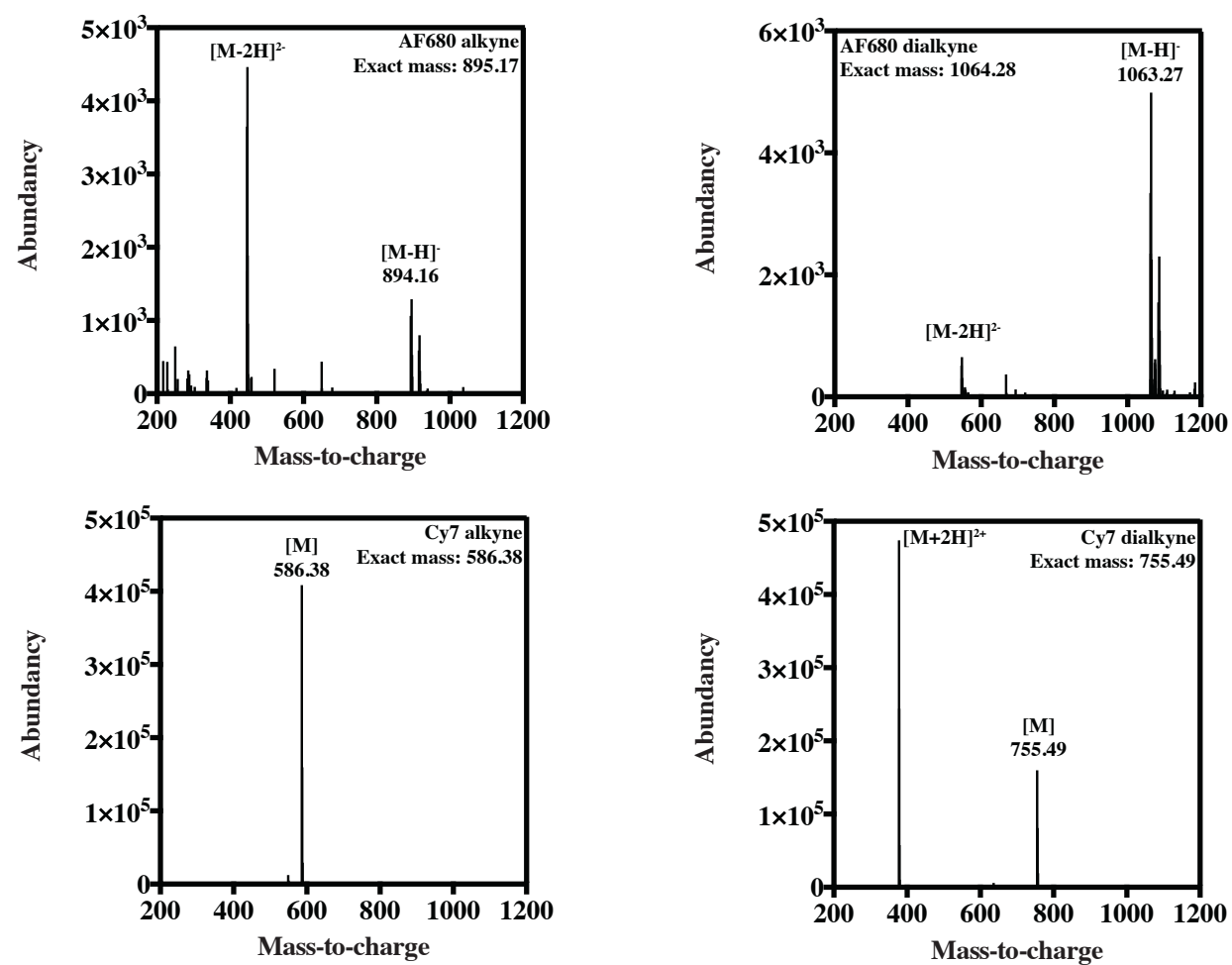

Figure S2. ESI spectra of alkyne functionalized fluorophores for peptide conjugation is shown above.

Fluorescent alkyne and dialkyne linkers were purified using RP-HPLC $(12 \mathrm{~mL} / \mathrm{min}$ flow rate) using linear gradients of water with $0.1 \%$ TFA (A) and acetonitrile with $0.1 \%$ TFA (B).

AF680 alkyne: $10-60 \%$ B 0.1-16 min; $t_{R}=12.1 \mathrm{~min}$.

AF680 dialkyne: $10-60 \%$ B 0.1-16 min; $t_{R}=11.7 \mathrm{~min}$.

Cy7 alkyne: 70\% B $0.1-15 \mathrm{~min} ; \mathrm{t}_{\mathrm{R}}=11.3 \mathrm{~min}$.

Cy7 dialkyne: 50\% B 0.1-4 min, 50-80\% B 4-20 min; $t_{R}=14.0$ min. 

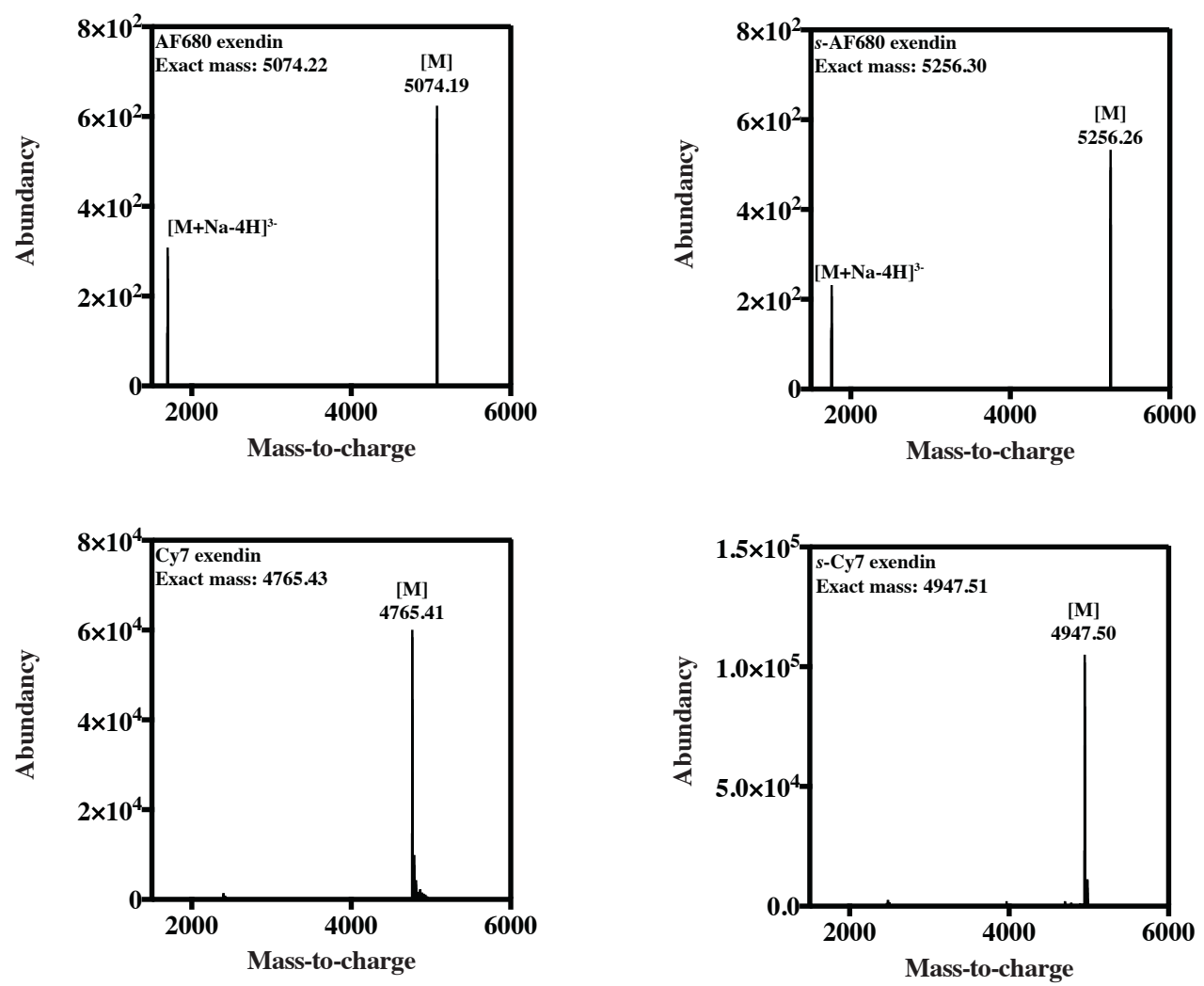

Figure S3. ESI spectra of fluorescent exendin conjugates are shown above.

Peptides were purified using RP-HPLC using linear gradients of water with $0.1 \%$ TFA (A) and acetonitrile with $0.1 \% \mathrm{TFA}(\mathrm{B})$ at $1 \mathrm{~mL} / \mathrm{min}$.

AF680 exendin: $20-50 \%$ B 0.1-24 $\mathrm{min} ; \mathrm{t}_{\mathrm{R}}=21.4 \mathrm{~min}$. $s$-AF680 exendin: $20-50 \%$ B 0.1-24 $\mathrm{min} ; \mathrm{t}_{\mathrm{R}}=20.1 \mathrm{~min}$. Cy7 exendin: $30-80 \%$ B $0.1-20$ min; $t_{R}=11.1$ min. $s$-Cy7 exendin: 30-80\% B 0.1-20 min; $\mathrm{t}_{\mathrm{R}}=11.7 \mathrm{~min}$.

Post-purification, stabilized peptides were reacted with excess fluorescent AlexaFluor 647 alkyne to verify no unreacted azides were present. HPLC and MALDI-TOF indicated no new species were formed. Isotopic spacing for deconvoluted peptide ESI spectra indicates no multimerization of peptide species. 


\section{HPLC Purity and Characterization}
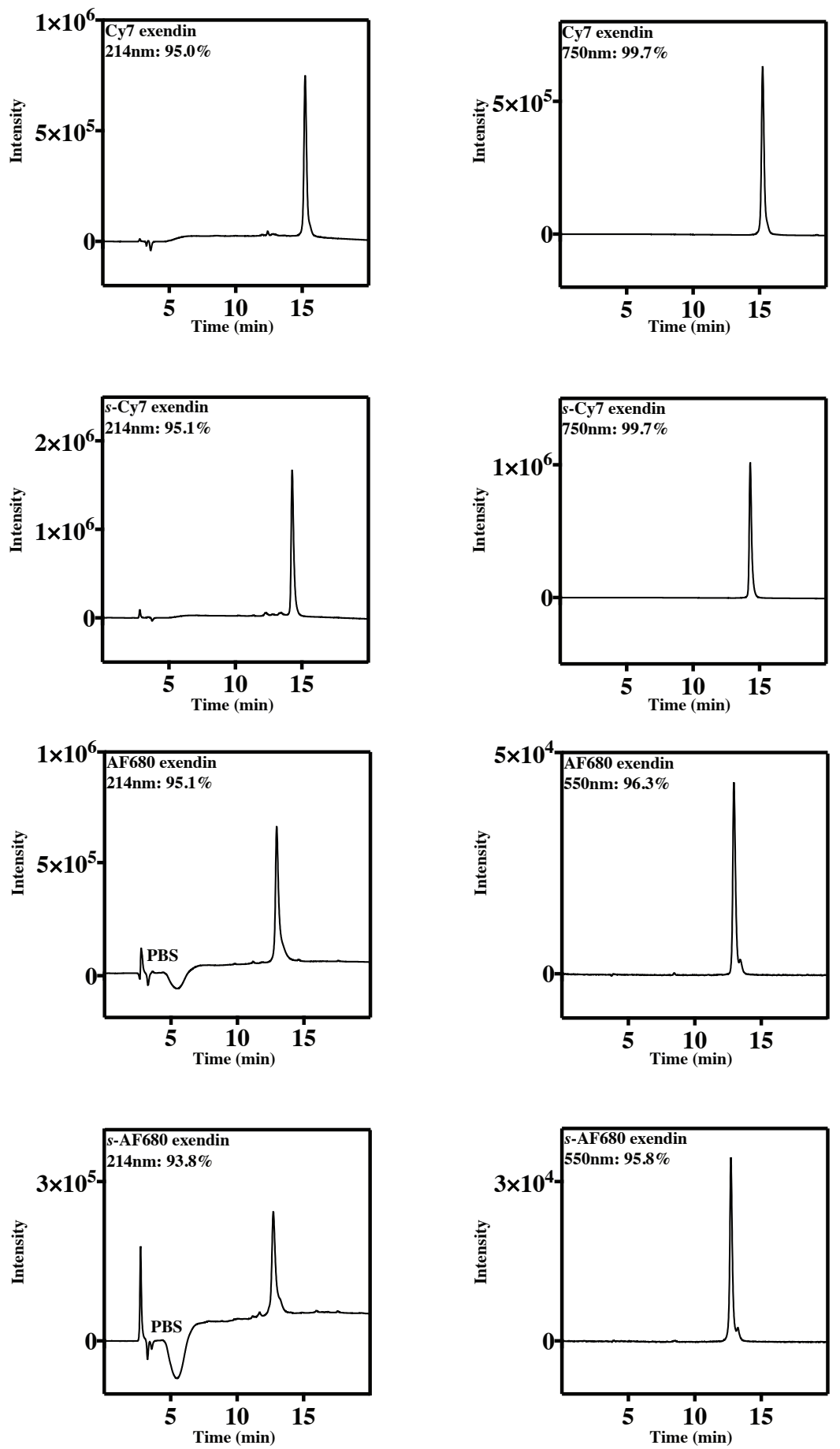

Figure S4. HPLC traces for fluorescent exendin conjugates demonstrating high purity at $214 \mathrm{~nm}$ and fluorophore absorbance wavelengths. Purity check method is 10-70\% B 0.1-19 min. 
In the presence of $0.1 \%$ TFA added to the mobile phase, AlexaFluor 680 absorbance max shifts from $680 \mathrm{~nm}$ to $\sim 550 \mathrm{~nm}$, hence detection at $550 \mathrm{~nm}$ on HPLC. This change in absorbance is reversible once TFA is removed/neutralized. Cy7 peptides are monitored at $750 \mathrm{~nm}$. Stabilized/non-stabilized conjugate purity evaluated at both fluorophore absorbance and $214 \mathrm{~nm}$ absorbance. There was no statistically significant difference in fluorescence intensity between stapled and non-stapled conjugates using either $\mathrm{Cy} 7$ or AF680 using a dilution series.

Absorbance spectra of fluorescently stabilized/non-stabilized peptides indicate no changes in maximum excitation compared to unreacted fluorophore. Compounds are dissolved in 1:1 water:acetonitrile.
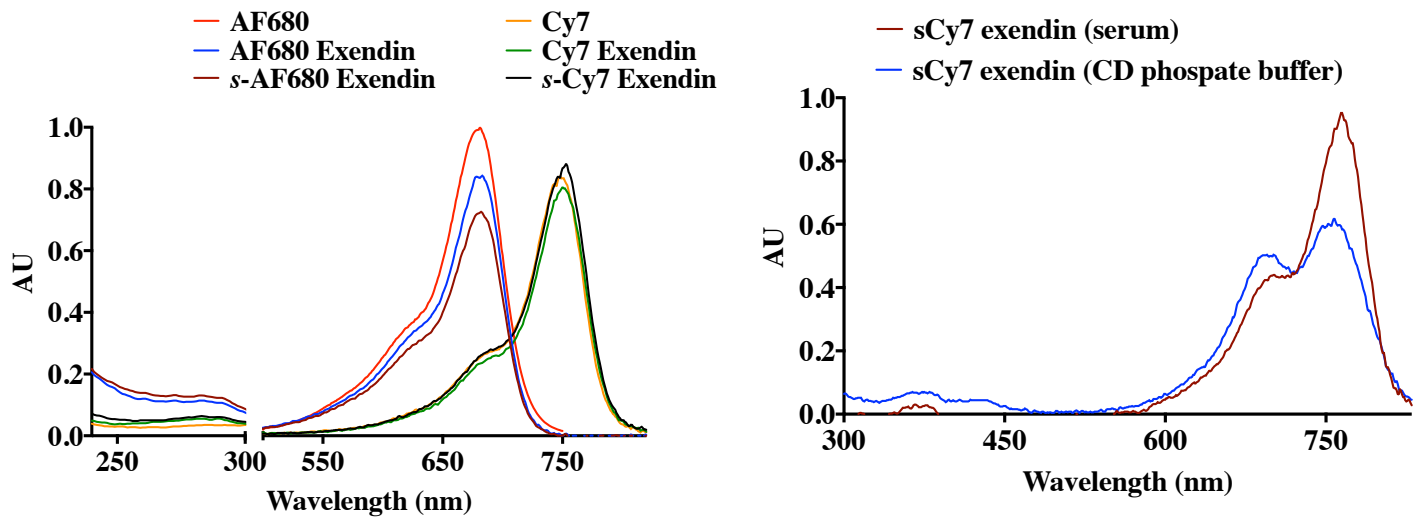

Figure S5. Absorbance spectra for exendin conjugates. (Left): absorbance profiles are as expected in 1:1 acetonitrile water; (right): for Cy7 conjugates, absorbance in $5 \mathrm{mM}$ potassium phosphate buffer shows a much stronger shoulder, indicative of $\mathrm{H}$-aggregates of Cy7 at these high concentrations ${ }^{1}$.

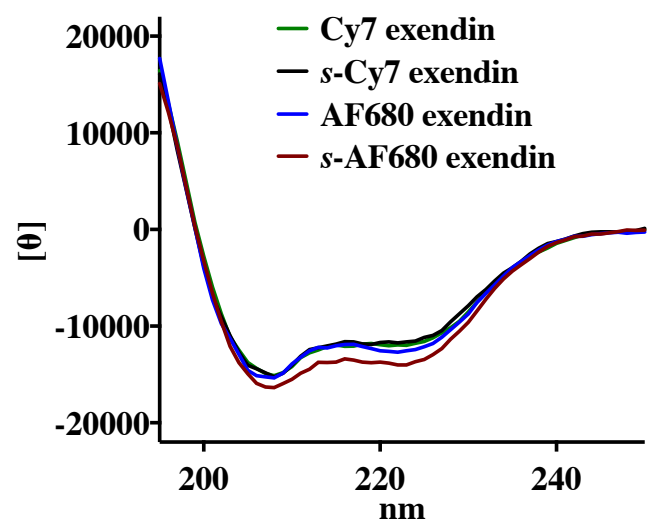

Figure S6. CD spectra for exendin conjugates collected in $5 \mathrm{mM}$ potassium phosphate buffer at room temperature. $\chi^{\text {helix }}$ for $s$-AF680, AF680, $s$-Cy7, and Cy7 exendin are 0.65, 0.59, 0.55, and 0.56, respectively. 


\section{Protease Digest Data}
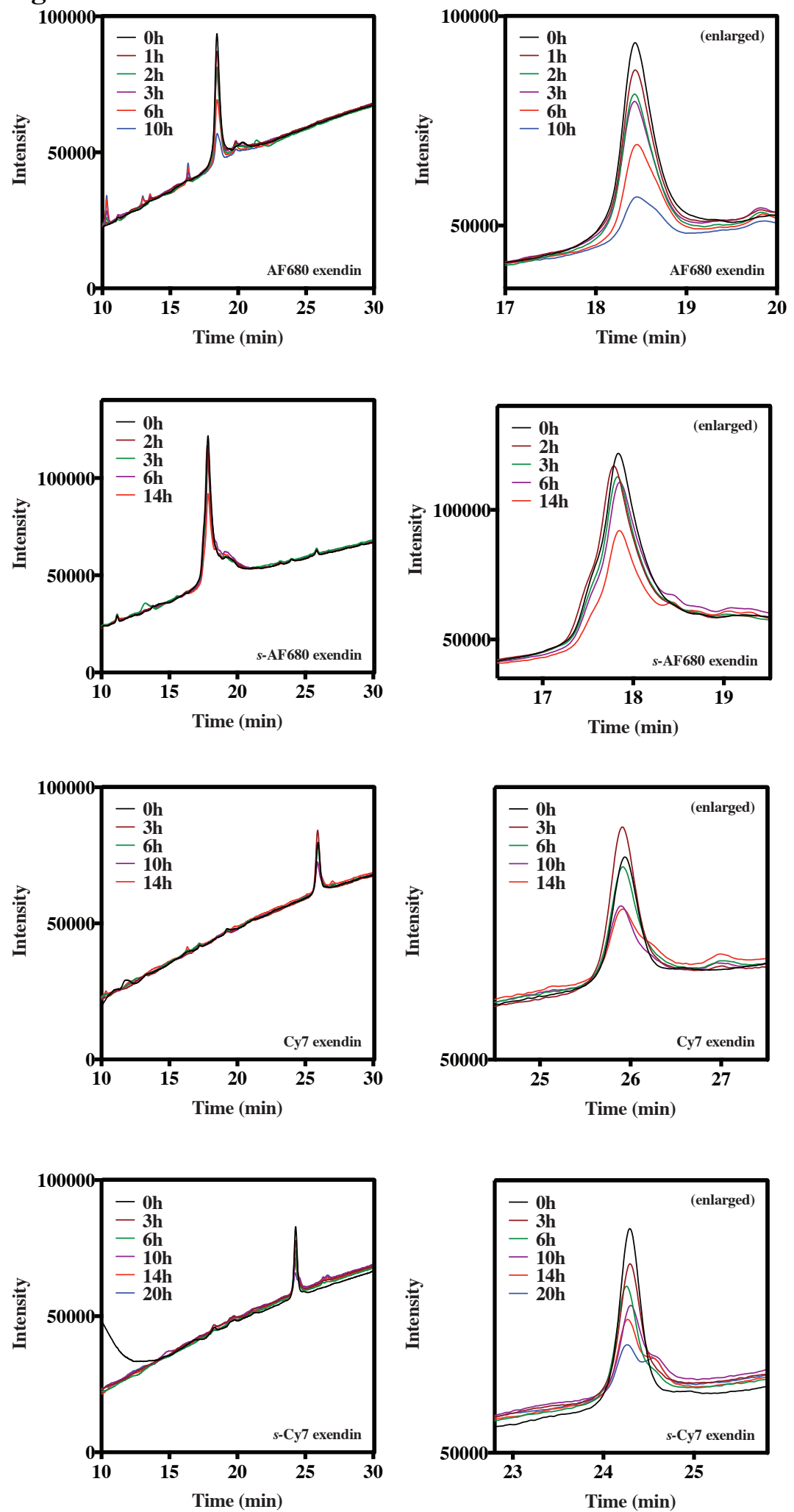

Figure S7. Peptide digests in trypsin were analyzed on RP-HPLC with $214 \mathrm{~nm}$ detection. Characteristic chromatograms are shown above. 
Gradient used for analysis were all the same: B (0.1\% TFA in acetonitrile), 20-60\%: 28 min. Unreacted double mutant exendin was subject to the same digest conditions as well. The results indicated the unreacted double mutant exendin digested with a half-life of $0.8 \pm 0.2$ $\mathrm{h}$, consistent with our previous findings. Interestingly, the digest of $s$-AF680 exendin in this manuscript is significantly slower than our previously published values. This is likely due to multiple reasons including an increase in overall stapled peptide purity in the current digest as well as differences in post-purification work-up of the stapled peptide. Previously, stapled peptides were concentrated on a rotary evaporator. The acidic $\mathrm{pH}$ resulting from residual TFA was then adjusted to $\mathrm{pH} 7.4$ with sodium bicarbonate. All exendin conjugates were co-rotavapped with methanol $3 x$ to remove residual TFA and then lyophilized. The current methods as reported in this manuscript do not require any additional $\mathrm{pH}$ adjustment steps. The $\mathrm{pH}$ of the reaction digest mixture was measured to confirm the appropriate $\mathrm{pH}$. Digests were performed in triplicate with the exception of the double mutant unreacted peptide, which was digested once to compare with previously published digests in triplicate.
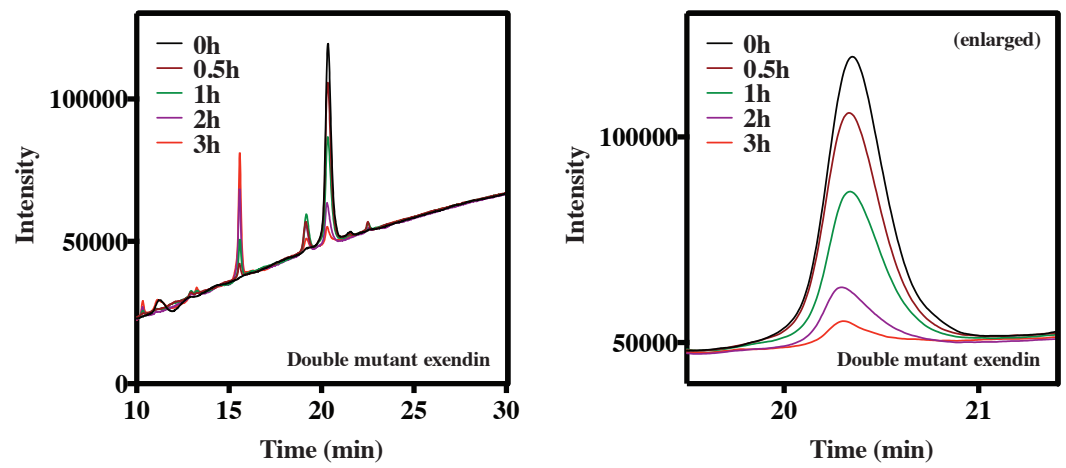

Figure S8. HPLC traces for digested double mutant exendin. Digest is rapid without helix stabilization.

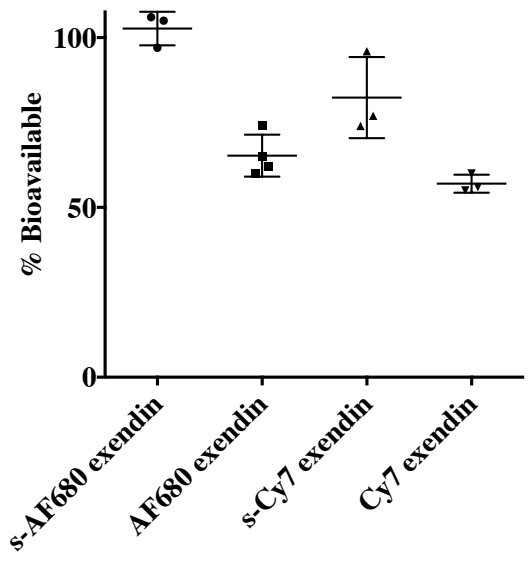

Figure S9. Unpaired t-test to confirm statistically significant differences for bioavailability between stapled and unstapled peptides. 


\begin{tabular}{|c|c|c|c|}
\hline Compound & AUC IV & AUC SC & \% Bioavailable \\
\hline$s$-AF680 exendin & $7330( \pm 300)$ & $7570( \pm 300)$ & $103( \pm 6)$ \\
\hline AF680 exendin & $5270( \pm 400)$ & $3430( \pm 200)$ & $65( \pm 6)$ \\
\hline$s$-Cy7 exendin & $19440( \pm 800)$ & $15850( \pm 2200)$ & $82( \pm 12)$ \\
\hline Cy7 exendin & $62100( \pm 10000)$ & $35370( \pm 1000)$ & $57( \pm 9)$ \\
\hline
\end{tabular}

Table S10. AUC data for all injected peptides including IV and SC injections.

SDS-page for exendin conjugates incubated in fresh serum and previously frozen plasma at $37^{\circ} \mathrm{C}$.

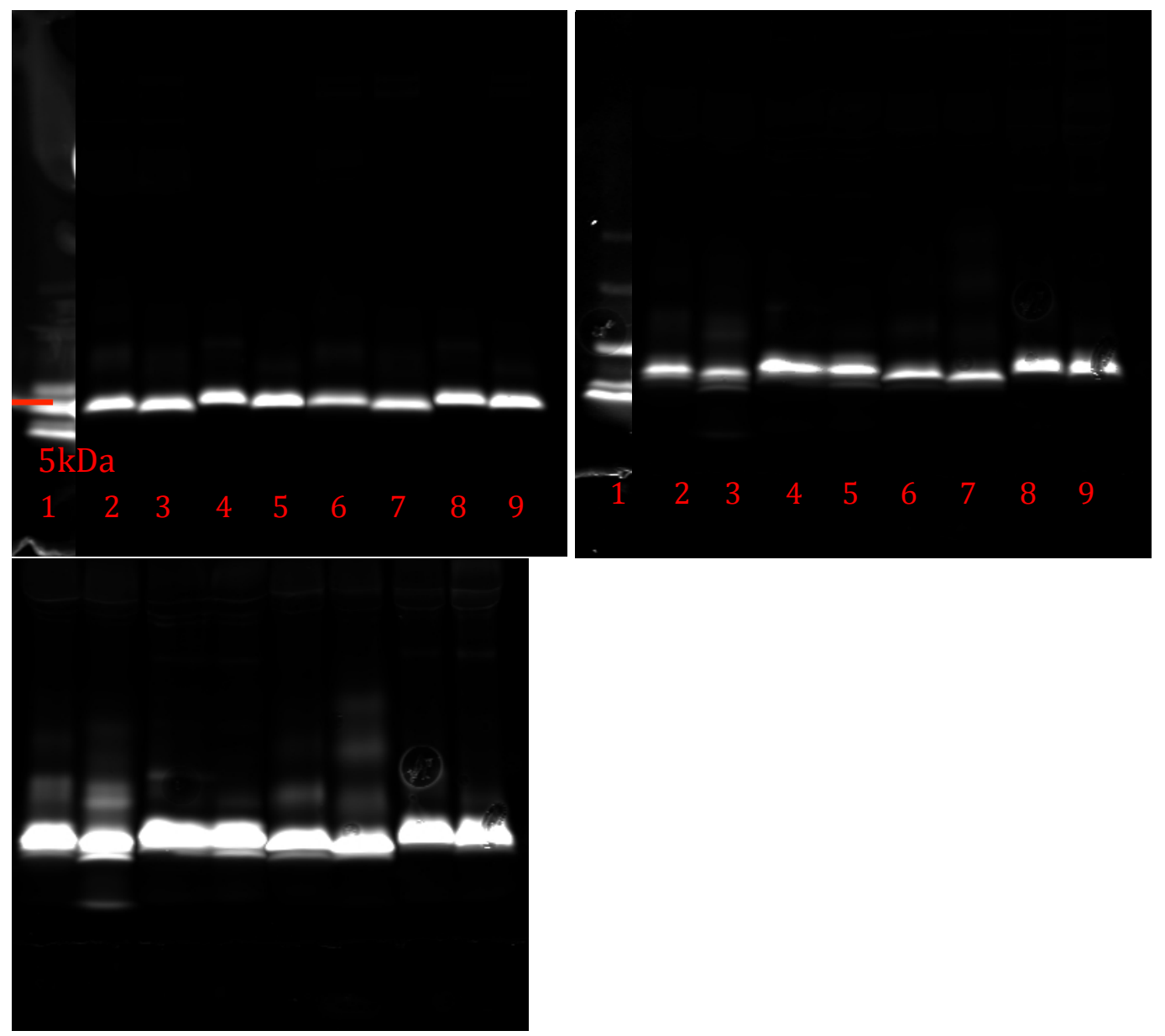

Figure S11. Left, top: $0 \mathrm{~h}$ incubation; right, top: $24 \mathrm{~h}$ incubation; left, bottom: $24 \mathrm{~h}$ incubation (window leveled to bring out fragments). Fluorescent values indicate little degradation of all conjugates in either fresh mouse serum or frozen plasma at $37^{\circ} \mathrm{C}$ over $24 \mathrm{~h}$. The ladder has been window leveled differently from the other lanes for ease of visualization. 
Fig. S11 Lane ID

1. Ladder

2. $s$-680 exendin (serum)

3. 680 exendin (serum)

4. $s$-Cy7 exendin (serum)

5. Cy7 exendin (serum)

6. $s$-680 exendin (plasma)

7. 680 exendin (plasma)

8. $s$-Cy7 exendin (plasma)

9. Cy7 exendin (plasma)
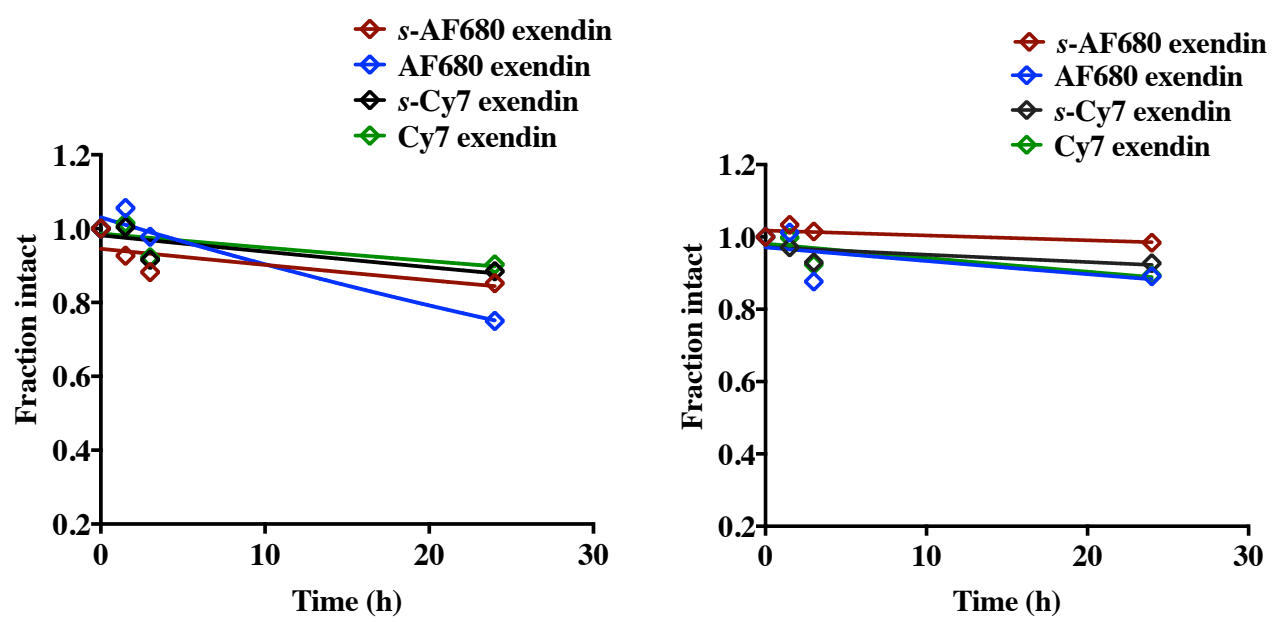

Figure S12. Serum (fresh) and mouse plasma stability data for all fluorescent exendin conjugates. Regions of interest were drawn on protein gels (S11) and fluorescence intensity was used to quantify fraction of intact peptide. All conjugates display slow digest half-lives in fresh mouse serum and frozen mouse plasma with only unstabilized AF680 peptide in fresh serum (isolated by cardiac puncture) showing more than $20 \%$ degradation over $24 \mathrm{~h}$. 


\section{In Vivo Stability Data}

SDS-page for plasma samples collected from SC injections via retro-orbital sampling
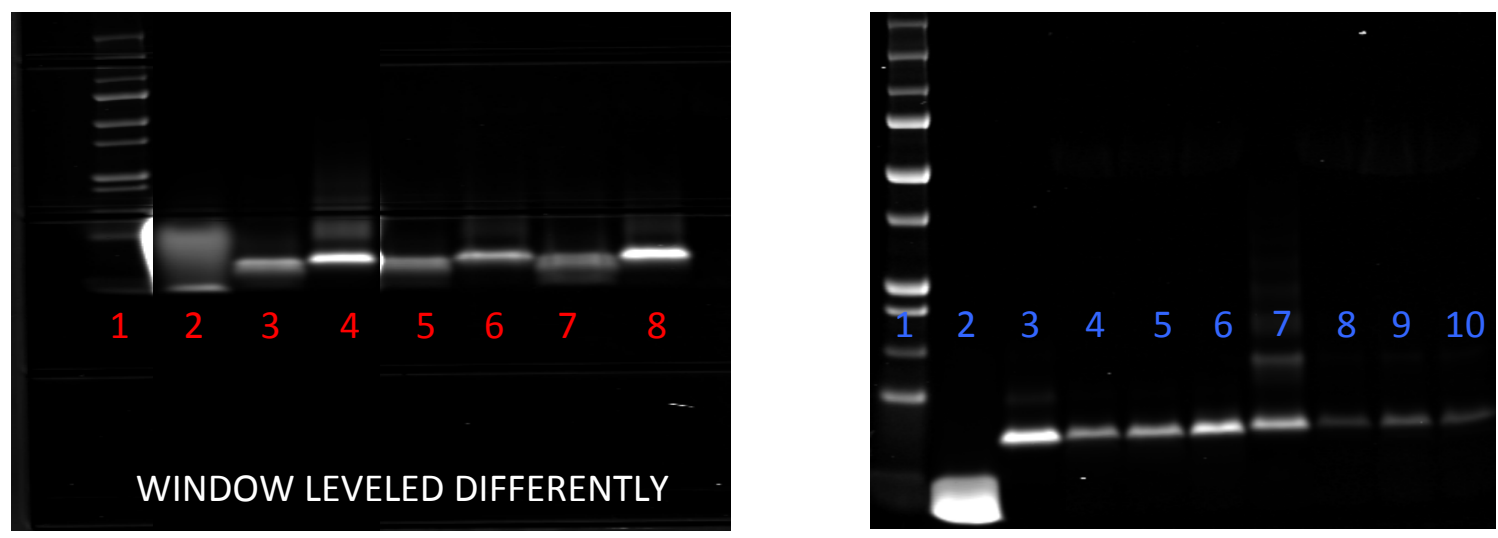

Figure S13. SDS-PAGE for plasma samples collected from SC injections. The protein gels (above) indicate plasma fluorescence intensity, which is used to calculate bioavailability, is primarily from intact peptide.

Gel (left): Lane ID

1. Ladder

2. AF680 dialkyne (free fluorophore, not injected)

3. 680 exendin (not injected)

4. $s$-680 exendin (not injected)

5. 680 exendin (30 $\mathrm{min})$

6. $s-680$ exendin $(30 \mathrm{~min})$

7. 680 exendin $(1 \mathrm{~h})$

8. $s$-680 exendin $(1 \mathrm{~h})$

Gel (right): Lane ID

1. Ladder

2. Сy7 (free fluorophore, not injected)

3. Cy7 exendin (not injected)

4. Cy7 exendin (30 min)

5. Cy7 exendin (1 h)

6. Cy7 exendin $(2.5 \mathrm{~h})$

7. s-Cy7 exendin (not injected)

8. $s$-Cy7 exendin (30 min)

9. $s$-Cy7 exendin $(1 \mathrm{~h})$

10. $s$-Cy7 exendin $(2.5 \mathrm{~h})$ 


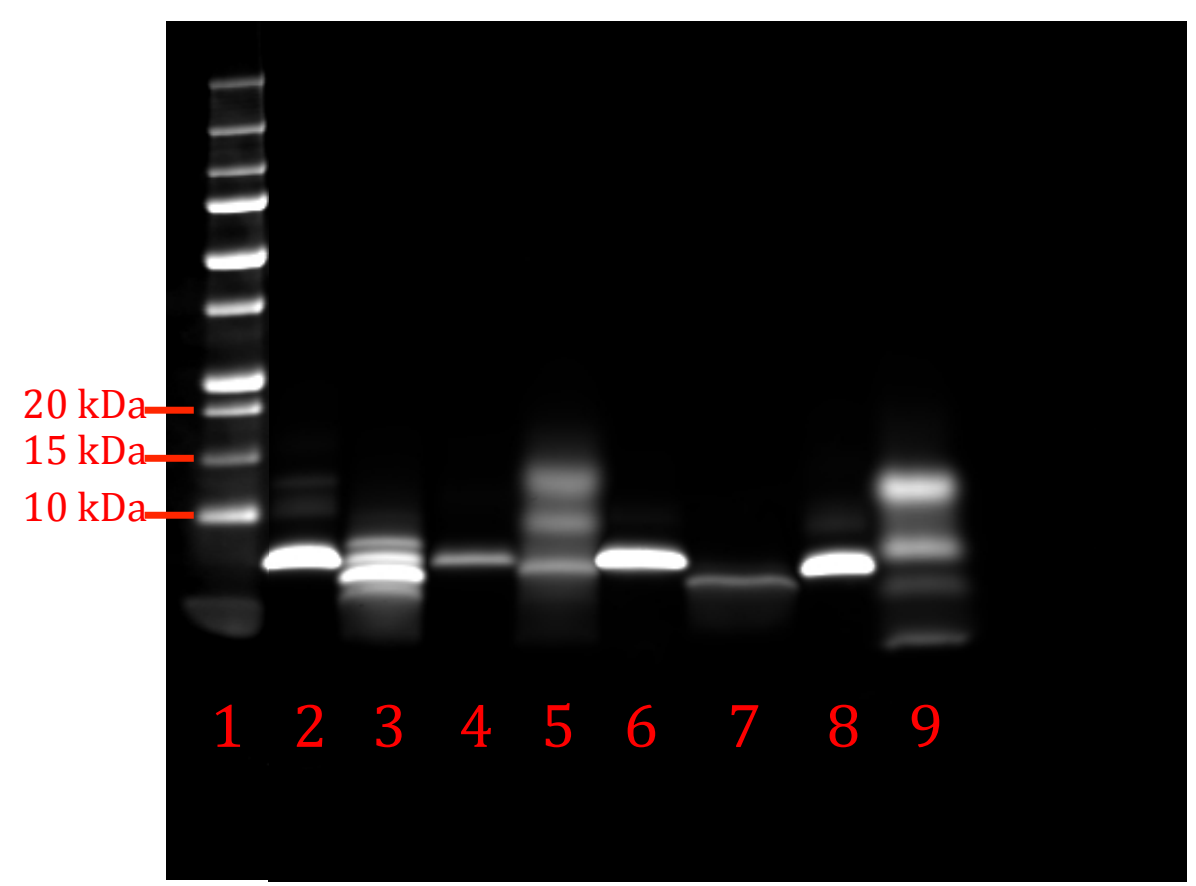

Figure S14. SDS-PAGE for digested samples demonstrating the appearance of new bands resulting from proteolytic degradation.

Lane ID

1. Ladder

2. $s$-Cy7 exendin (undigested)

3. $s$-Cy7 exendin (digested)

4. $s-680$ exendin (undigested)

5. $s$-680 exendin (digested)

6. Cy7 exendin (undigested)

7. Cy7 exendin (digested)

8. 680 exendin (undigested)

9. 680 exendin (digested)

To verify that stapled peptides display a qualitative difference on SDS-page when digested with trypsin, all the fluorescent peptide conjugates were digested and run on a protein gel. As expected, the digested sample lanes show remarkably difference profiles compared to non-digested samples. The ladder-containing lane is window-leveled differently to visualize the band intensity. Cy7 exendin (lane 6,7) is qualitatively the most similar of all the conjugates when comparing digested and non-digested peptide. SDS-fluorophore interactions do not necessarily follow the MW indicated by the ladder. 


\section{Model and Fits of Subcutaneous Delivery}

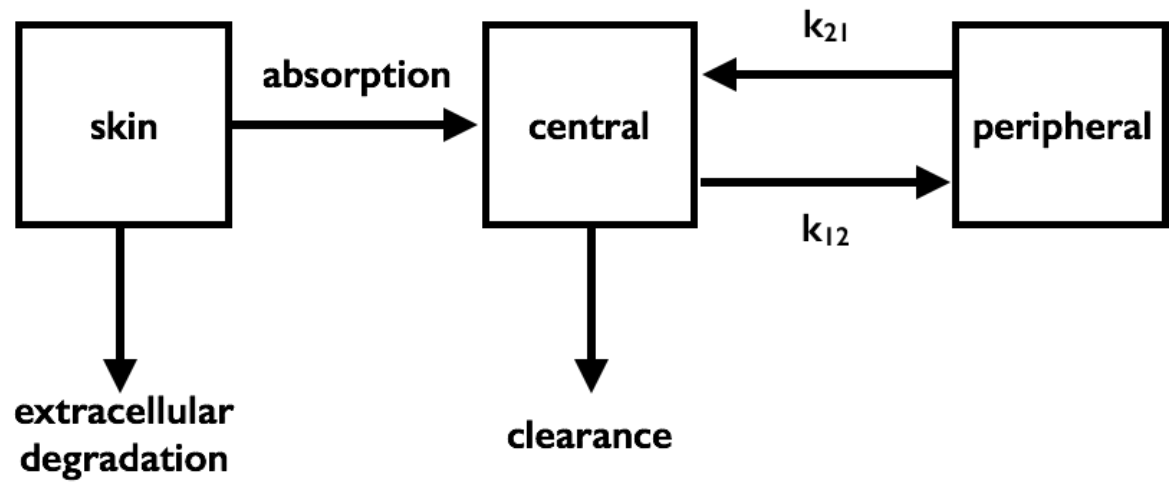

Figure S15. Three-compartment model illustrating biological processes affecting the SC absorption process, namely protease degradation.

A: skin

B: central compartment

C: peripheral compartment

$\mathrm{k}_{\mathrm{abs}}$ : first order absorption, $\mathrm{min}^{-1}$

$\mathrm{k}_{\mathrm{deg}}$ : first order degradation, $\min ^{-1}$

$\mathrm{V}_{\mathrm{A}}$ : skin compartment volume, $\mathrm{L}$

$\mathrm{k}_{\mathrm{cl}}$ : clearance from central compartment, $\min ^{-1}$

$\mathrm{k}_{12}$ : transport from central to peripheral, min $^{-1}$

$\mathrm{k}_{21}$ : transport from peripheral to central, $\mathrm{min}^{-1}$

$\mathrm{C}_{\mathrm{B}}=$ central compartment concentration (measured for each peptide), $\mathrm{nM}$

$\mathrm{C}_{\mathrm{c}}=$ peripheral compartment concentration, $\mathrm{nM}$

$\mathrm{X}_{\mathrm{A}}=$ moles in skin compartment, nmole

$\mathrm{X}_{\mathrm{A}, 0}=$ injected SC dose, 1 nmole

$\mathrm{X}_{\mathrm{B}, 0}=0$

$\mathrm{X}_{\mathrm{C}, 0}=0$

$\mathrm{k}_{\alpha}=$ alpha clearance (biexponential)

$\mathrm{k}_{\beta}=$ beta clearance (biexponential)

$\mathrm{f}_{\mathrm{A}}=$ fraction fast (fit from biexponential)

Balance on skin compartment:

$\frac{d X_{A}}{d t}=-k_{a b s} X_{A}-k_{\operatorname{deg}} X_{A}$ with the initial condition, $\mathrm{t}=0, \mathrm{X}_{\mathrm{A}}=\mathrm{X}_{\mathrm{A}, 0}=1 \mathrm{nmol}$

$X_{A}=X_{A, 0} \exp \left(-\left(k_{a b s}+k_{\mathrm{deg}}\right) * t\right)$

Balance on central compartment:

$\frac{d C_{B}}{d t}=\frac{k_{a b s}}{V_{A}} * X_{A, 0} * \exp \left(-\left(k_{a b s}+k_{\mathrm{deg}}\right) * t\right)-k_{c l} * C_{B}-k_{12} C_{B}+k_{21} C_{C}$

Balance on peripheral compartment:

$\frac{d C_{C}}{d t}=k_{12} C_{B}-k_{21} C_{C}$

$\mathrm{k}_{21}, \mathrm{k}_{12}$, and $\mathrm{k}_{\mathrm{cl}}$ are calculated using the following relationships for biexponential decay: 
$k_{\alpha}+k_{\beta}=k_{c l}+k_{12}+k_{21}$

$k_{\alpha} * k_{\beta}=k_{c l} * k_{21}$

$f_{A}=\frac{k_{21}-k_{\alpha}}{k_{\beta}-k_{\alpha}}$

Experimental plasma concentrations $\left(C_{B}\right)$ are used to determine alpha and beta clearance half lives and are used as input to determine the rate constants between the central and peripheral compartments using Prism software. Using the injected dose of $1 \mathrm{nmol}\left(\mathrm{X}_{\mathrm{A}, 0}\right)$ and the experimentally determined bioavailability, the ODE for the central compartment was used to fit for $\mathrm{k}_{\mathrm{abs}}, \mathrm{k}_{\mathrm{deg}}$, and $\mathrm{V}_{\mathrm{A}}$ simultaneously. Each peptide was fit separately (Fig. S14) with absorption and degradation half lives summarized in Table 1.

\begin{tabular}{|c|c|c|c|}
\hline Compound & $\mathrm{k}_{\mathrm{abs}}\left(\mathrm{min}^{-1}\right)$ & $\mathrm{k}_{\operatorname{deg}}\left(\mathrm{min}^{-1}\right)$ & $\mathrm{V}_{\mathrm{A}}(\mathrm{mL})$ \\
\hline$s$-AF680 exendin & $0.0087( \pm 0.0018)$ & $0.00065( \pm 0.00026)$ & $1.3( \pm 0.06)$ \\
\hline AF680 exendin & $0.0077( \pm 0.0014)$ & $0.0041( \pm 0.00079)$ & $2.0( \pm 0.26)$ \\
\hline$s$-Cy7 exendin & $0.0059( \pm 0.0015)$ & $0.0013( \pm 0.00034)$ & $2.6( \pm 0.33)$ \\
\hline Cy7 exendin & $0.0036( \pm 0.0006)$ & $0.0029( \pm 0.00044)$ & $1.4( \pm 0.09)$ \\
\hline
\end{tabular}

Table S16. Fitted absorption, degradation and volume values from a 3-compartment model of subcutaneous absorption. Data from each mouse were fit separately. 
Matlab fitted curves from experimental SC data to determine first order absorption and degradation rate constants for the four exendin conjugates. Fits can be sensitive to initial guesses for rate constants so relative tolerance was decreased to $10^{-12}$ for fits.
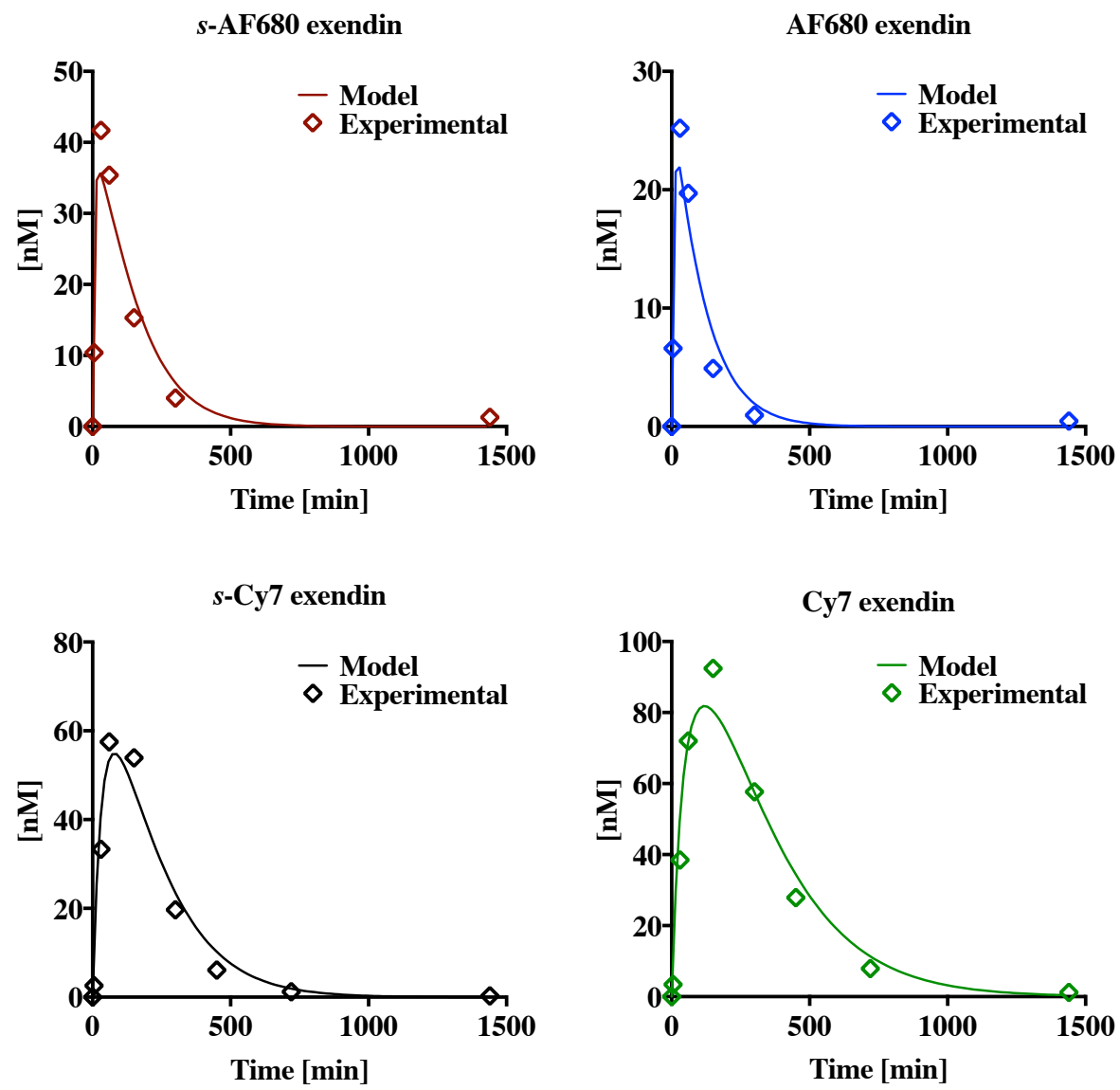

Figure S17. MATLAB fits for experimentally measured SC plasma concentration of fluorescent peptide. The fits are used to determine the degradation and absorption rates. 


\section{LogD Data}

LogD values were measured for all fluorescent exendin conjugates. Briefly, a $5 \mu \mathrm{M}$ solution of peptide in PBS was allowed to equilibrate with octanol at $37^{\circ} \mathrm{C}$ overnight. (PBS and octanol were pre-equilibrated prior to adding the probe.) The aqueous component was quantified using a NIR scanner to determine the amount of peptide in both phases, and the $\log \mathrm{D}$ values were calculated and listed in Table S17. While a more helical peptide may be expected to have a higher $\log \mathrm{D}$ (due to increased intramolecular hydrogen bonding), even the unstabilized exendin has a fairly large helicity. Therefore, the PEG linker, additional triazole, and tertiary amine on the stabilized linker-dye conjugate are likely responsible for the more hydrophilic stabilized peptide values.

\begin{tabular}{|c|c|}
\hline Compound & LogD \\
\hline$s$-AF680 exendin & $-0.48( \pm 0.2)$ \\
\hline AF680 exendin & $-0.32( \pm 0.03)$ \\
\hline$s$-Cy7 exendin & $0.005( \pm 0.03)$ \\
\hline Cy7 exendin & $0.60( \pm 0.01)$ \\
\hline
\end{tabular}

Table S18. Experimentally measured $\log \mathrm{D}$ values for exendin conjugates Cy7 conjugates are more lipophilic than AF680 conjugates. 


\section{Non-specific cellular uptake}

Non-specific uptake rates for the four fluorescent exendin conjugates were measured previously ${ }^{2}$ and shown in tabulated form below.

\begin{tabular}{|c|c|}
\hline Compound & $\begin{array}{c}\text { Non-specific uptake } \\
\text { rate }\left(\mathrm{s}^{-1}\right)\end{array}$ \\
\hline$s$-AF680 exendin & $1.63( \pm 0.02) \times 10^{-6}$ \\
\hline $\mathrm{AF680}$ exendin & $8.12( \pm 0.08) \times 10^{-7}$ \\
\hline$s$-Cy7 exendin & $1.65( \pm 0.04) \times 10^{-5}$ \\
\hline Cy7 exendin & $1.20( \pm 0.04) \times 10^{-4}$ \\
\hline
\end{tabular}

Table S19. Experimentally determine non-specific cellular uptake rates performed in media containing $10 \%$ serum.

AF680 cellular uptake rates are slow compared to in vivo SC absorption rates and likely do not play a role in determining the biodistribution of the peptides. Non-specific cellular uptake half-lives for Cy7 conjugates, namely non-stabilized Cy7 occur on similar time scales as SC absorption in the table listed above. (A $1.2 \times 10^{-4} / \mathrm{s}$ rate is equivalent to a $1.6 \mathrm{~h}$ half-life of cellular uptake.) Since the absorption rate results in a $3.2 \mathrm{~h}$ half-life, the would indicate cellular uptake could be significant. However, we hypothesized that the $10 \%$ serum in media would result in more probe sticking to cells that may be expected in vivo. To measure cellular uptake in the presence of a more physiological concentration of protein (e.g. albumin, where the interstitial concentration in the skin is $\sim 50 \%$ that of the serum, e.g. ${ }^{3}$ ), we repeated the cellular uptake assay in the presence of $50 \%$ serum.

When uptake assays were performed in 50\% serum in media, the non-specific uptake rates of stabilized and non-stabilized Cy7 exendin decreased to $8.9 * 10^{-6}$ and $1.7 * 10^{-5}$, respectively. The fastest cellular uptake rate therefore was much slower ( $28 \mathrm{~h}$ half-life) compared to the $3.2 \mathrm{~h}$ half-life of absorption. Due to these low non-specific uptake rates, it is unlikely that cellular uptake at the site of injection is significantly impacting the bioavailability. 


\section{References}

(1) Wurth, C., Grabolle, M., Pauli, J., Spieles, M., and Resch-Genger, U. (2013) Relative and absolute determination of fluorescence quantum yields of transparent samples. Nat Protoc 8, 1535-50.

(2) Zhang, L., Bhatnagar, S., Deschenes, E., and Thurber, G. M. (2016) Mechanistic and quantitative insight into cell surface targeted molecular imaging agent design. Scientific Reports 6.

(3) Worm, A. M. (1981) Exchange of macromolecules between plasma and skin interstitium in extensive skin disease. The Journal of investigative dermatology 76, 489-92. 